\title{
EchoGéo
}

41 | 2017

Un état des lieux du Brésil en 2017

\section{Archéologie de la question agraire au Brésil : du labor grabbing au land grabbing}

Cássio Arruda Boechat, Ana Carolina Gonçalves Leite and Carlos de Almeida Toledo

\section{OpenEdition}

1 Journals

\section{Electronic version}

URL: https://journals.openedition.org/echogeo/15112

DOI: 10.4000/echogeo.15112

ISSN: 1963-1197

\section{Publisher}

Pôle de recherche pour l'organisation et la diffusion de l'information géographique (CNRS UMR 8586)

\section{Electronic reference}

Cássio Arruda Boechat, Ana Carolina Gonçalves Leite and Carlos de Almeida Toledo, "Archéologie de la question agraire au Brésil : du labor grabbing au land grabbing", EchoGéo [Online], 41 | 2017, Online since 28 September 2017, connection on 31 July 2021. URL: http://journals.openedition.org/echogeo/ 15112 ; DOI: https://doi.org/10.4000/echogeo.15112

This text was automatically generated on 31 July 2021 .

EchoGéo est mis à disposition selon les termes de la licence Creative Commons Attribution - Pas d'Utilisation Commerciale - Pas de Modification 4.0 International (CC BY-NC-ND) 


\title{
Archéologie de la question agraire au Brésil : du labor grabbing au land grabbing
}

\author{
Cássio Arruda Boechat, Ana Carolina Gonçalves Leite and Carlos de \\ Almeida Toledo
}

\section{Introduction}

1 La littérature sur la question agraire brésilienne s'appuie sur un dualisme qui met en opposition les aspects d'un retard et les possibilités de «notre » modernisation, aussi nous avons senti le besoin d'une critique de ce dualisme. Ainsi, notre parcours devra repenser le concept de « région » et de son incorporation à la planification menée par l'État. Cela découle de la préoccupation de classer la colonisation et la constitution de régions pour ne pas retomber sur des caractérisations basées sur des oppositions à la contemporanéité. Nous proposons alors deux questions à prendre en compte. La première, à partir de l'affirmation que la modernisation est fondée sur une autonomisation des catégories de terre, travail, État et capital, auparavant confondues en représentations qui en réunissaient plusieurs. La deuxième propose que la question agraire soit développée à partir de la question régionale qui émerge entre agriculture et industrie, et entre rural et urbain, sans négliger la nécessité de reprendre des éléments qui conditionnent la formation de la première. Puisque nous ne croyons pas que de telles discussions soient datées, avec des positions dualistes qui répondent dans la production récente sur le "monde rural», nous réaffirmons le besoin de cette critique. Notre " archéologie » cherche à trouver, ainsi, le sens d'un cadavre qui hante la question agraire contemporaine.

2 Il est possible de mettre en rapport ces questions quand nous pensons que l'autonomisation du capital implique un déploiement de segments du marché, de façon à consolider un marché foncier, un marché du travail et un autre des éléments du capital et de l'argent, à portée nationale, séparés entre eux et séparés de l'État. 
L'industrialisation suppose alors la constitution du marché de capitaux et demande l'approvisionnement en matières-premières, en travailleurs disponibles, outre l'intermédiation du crédit pour dynamiser les investissements, l'État en position de médiateur de ses liens les plus divers. Cependant, nous retenons l'idée selon laquelle l'autonomisation n'est pas l'autonomie, la valorisation du capital continuant à dépendre de l'exploration des travailleurs, sans qu'il y ait, comme dans les autres catégories (terre, capital et État) d'auto-rémunération possible sans ce prérequis, même si cela se passe dans des lieux autres que celui où se trouve l'élément rémunéré. Ce développement des différents marchés constitue, en même temps, la généralisation d'une forme sociale unique passant, par le marché et par la diminution des personnes, des lieux et des choses à la qualité générique de marchandises ou de leurs propriétaires. Nous pouvons alors traiter l'autonomisation également comme un processus d'homogénéisation de cette forme sociale fétichiste. La paire hétérogénéité/ homogénéité organise les idées qui s'en suivent, en évitant de certifier dans la hâte la prévalence d'un des pôles. L'introduction faite, passons à la critique proprement dite.

\section{Question régionale et question agraire}

Il n'est certes pas nouveau de dire que « région » est un concept se trouvant à la base de la caractérisation de la géographie traditionnelle, surtout celle inspirée des auteurs français de la fin du XIX siècle et de la première moitié du XX ${ }^{e}$, traitée comme une synthèse particulière $d u$ rapport entre l'homme et l'environnement, devant être décrite sur un mode monographique, et que ce concept et la méthode ont fait l'objet de critiques diverses à partir des années 1950/1960. Nous allons reprendre ici, néanmoins, une approche qui a repensé de façon critique le concept de région sans l'abandonner, le dessinant comme l'élément particulier d'une totalité contradictoire ou une partie de la division internationale du travail, en incluant dans sa caractérisation une dimension importante du pouvoir ou de la domination apparemment politique.

4 F. de Oliveira (2008) a proposé une approche de la soi-disant question régionale contribuant ainsi à l'organisation d'une histoire de controverses interprétatives et des processus sociaux qui ont mené au conditionnement du débat autour de la question agraire brésilienne. Il a indiqué une voie de modernisation qui rompait avec les régions tout en instaurant une tendance à l'homogénéisation des rapports sociaux de production, celle-ci aboutissant à délimiter seulement des inégalités régionales, de façon à constituer des "zones de localisation différentielle» comme dans le développement qu'il a repéré aux EUA :

« Il y a une tendance à la complète homogénéisation de la reproduction du capital, menant à la disparition des 'régions', dans le sens proposé par cet examen. [...] Une telle tendance ne réussit presque jamais à se matérialiser sur un mode complet et fini, de par le fait même que le processus de reproduction est, par définition, inégal et combiné, mais dans certains espaces du monde capitaliste, dont peut-être l'économie américaine est l'exemple le plus complet, le degré d'homogénéisation procuré par la concentration et par la centralisation du capital est indéniable, de façon à rendre presque totalement floues les différences entre les segments divers du territoire national nord-américain » (Oliveira, 2008, p. 146).

Il décrit alors l'incidence de la modernisation sur des régions rurales en tant qu'une dynamique homogénéisante. Il nous appartiendrait de poser la question de savoir quels contenus étaient modifiés et selon quels critères la dynamique imposée les égalait. En 
quelques mots, qu'est-ce que la région ? Comment et pourquoi change-t-elle ? Qu'est-ce qui caractérise l'espace résultant de l'autonomisation? Reprendre ces questions nous permet d'introduire les présupposés autour des débats sur la question agraire. Le lecteur attentif comprendra alors que la même question revient aujourd'hui avec de nouveaux traits : qu'est-ce le (nouveau) rural ? Comment et pourquoi se transforme-til ? (voir Buainain et al., 2014 ; Wanderley, 2013 ; Carneiro, 2008; entre autres). Partons donc de la réponse de cet auteur :

«L"enfermement' d'une région par ses classes dominantes demande, exige et ne se présente, donc, que tant que ses classes dominantes sont à même de reproduire le rapport social de domination, ou, plus clairement, les rapports de production. [...] L'ouverture' de la région et la conséquente 'intégration' nationale, au cours du long parcours jusqu'à la complète dissolution des régions, se manifeste quand le rapport social ne peut plus être reproduit et, face à cette impossibilité, filtre la perte de l'hégémonie des classes dominantes locales et leur substitution par d'autres, de caractère national et international » (Oliveira, 2008, p. 151-152).

6 Nous voyons ici une définition qui qualifie la région de façon distincte de celle de la géographie régionale, de par sa difficulté de définition. D'où la suggestion «d'une forme spéciale de reproduction du capital» (Oliveira, 2008, p. 151) - suggérant cependant une conformation territoriale ayant, au fond, le sens d'éviter la libre «mobilité du travail» (Gaudemar, 1977), comme une armure - armature, selon la définition de Lipietz (1988) dans $O$ capital e seu espaço, où il théorise le concept de façon similaire (voir Seabra et Goldestein, 1982) - qui « ferme » politiquement la région, en présupposant des tensions intra et inter régionales, l'État national étant en formation.

7 En ce qui concerne le contenu social de la région, il y aurait une réalité qui, étant présupposées les catégories scindées de la pensée, pourrait être qualifiée en tant que fusionnant les pouvoirs économique et politique (Oliveira, 2008); ou les sphères privée et publique (Leal, 1976); ou combinant violence extra-économique et violence du marché (Rangel, 1986). Dans une lecture plus étendue, nous pourrions repenser les modes par lesquels seraient caractérisés les «blocages » au libre exercice de la mobilité du travail au-delà de la simple contrainte physique, si bien que les rapports sociaux établis dans une région impliqueraient, comme cela a été proposé par Franco (1997) les éléments d'une domination personnelle incluant lien de famille et parenté politique, ainsi que le «colonelisme», souligné en sociologie (Queiroz, 1975). Tandis que Holanda (1995) avait observé la proéminence de la personne sur les lois impersonnelles caractérisant la « cordialité » qui renvoyait au primat de la «faveur » sur le « contrat » (Schwarz, 2000), Faoro (1989) proposait la théorie de l'institution du «patrimonialisme» dans lequel propriété et structure foncières fondaient la sociabilité.

8 En effet, l'accès partiel et médiat au moyens de production par les travailleurs, largement caractérisés comme des "agrégés ", mais assumant, dans chaque région, des formes particulières (colon, habitant, cowboy, etc.), articulé à l'accès aux rapports personnels avec les fermiers et aux capacités de contrainte centrées sur ces derniers, données par l'État, composaient un scénario de définition difficile. Pour Rangel (1986), il s'agissait d'un féodalisme traditionnaliste. Martins (1998) et Oliveira (1987) observaient des relations non spécifiquement capitalistes qui renvoyaient la paysannerie à une situation postcoloniale. Sur un autre mode, pour Prado Jr (1979), Brant (1976) et D'Incao (1979), il y avait des formes d'emploi déguisées, le partenariat ou le métayage baissant les coûts de la production en temps de crise. Furtado (2000), à 
son tour, distingue trois secteurs de l'économie nationale: subsistance, exportation agraire et manufacture. La neutralité apparente de la distinction, toutefois, révélait son souci avec la « régression économique » des régions pendant les crises, quand celles-ci perdaient leur dynamisme provoqué par l'insertion au marché.

9 Suite à ces propositions, s'ouvre un débat national controversé sur un concept cher à la géographie traditionnelle. Pour la géographie régionale française, la région apparaissait comme un "genre de vie» (Lencioni, 1999). Pour cette raison, Monbeig (1998), dans sa thèse sur la culture du café à São Paulo, cherche des données pour vérifier la constitution d'une région: une relation particulière à l'environnement, celui-ci étant transformé de paysage naturel en culturel; un sentiment d'appartenance ; une stabilité relative des relations sociales. Même si cette géographie était au service des propos de la colonisation et de l'intégration nationale, en cartographiant les ressources et les potentialités économiques des territoires (Moraes, 2003), nous observons la conception d'un regard qui cherche l'hétérogénéité, dans un cadre diversifié de "genres de vie» dans leur rapport écologique (Sorre, 1998) à l'environnement. Cependant, surtout à partir des lectures des intellectuels de 1930 et de ceux qui les ont suivis (Jackson, 2007), cette hétérogénéité est classée en tant que forme particulière de domination sociale, résultant en une question régionale, traitée soit par le regard de ses inégalités, soit par ses entraves à la modernisation (Oliveira, 1993).

Dans d'autres travaux (Toledo, 2008 ; Toledo et Boechat, 2012 ; Boechat, 2014 ; et Leite, 2015), nous avons pu reconsidérer le dualisme inhérent à la dichotomie retard/ moderne comme située historiquement dans l'inflexion donnée à la modernité par les standards de sociabilité de l'État moderne, originaire de la Révolution française, et par les standards de productivité du capital, originaire de la Révolution industrielle anglaise. Cette proposition a traversé la relecture des classiques mentionnés ci-dessus, en évitant leurs dualismes et profitant des éléments permettant la compréhension de rapports particuliers. Ainsi, la perspective adoptée ici établit un concept de région qui n'est ni celui opposé de façon péjorative à l'industrie et à la métropole, ni celui de la perspective supposément neutre de la planification étatique - matérialisée par l'Instituto Brasileiro de Geografia e Estatística (IBGE) et, au cours d'une longue période, influencée par une certaine géographie quantitative. Et encore moins dans l'intention de reprendre le concept-clé de la géographie traditionnelle, pensé en tant que genre de vie situé dans une région naturelle spécifique. Nous aboutissons ainsi à la réévaluation de formules classiques sur la question régionale devant être discutées dans une question agraire du Brésil. Nous suggérons un concept qui renvoie au processus contradictoire de territorialisation des rapports sociaux de production capitaliste qui, au Brésil, nous réclame l'inclusion dans le débat sur la colonisation de l'Ancien Régime.

\section{Labor grabbing en tant que fondement de la colonisation dans l'Ancien Régime}

11 Revenant à Prado Jr. (2000) et Novais (2005), le Brésil colonial a été moderne dès le début, car intégré à l'expansion du marché européen et comme une entreprise de l'État absolutiste portugais. L'explication du «sens de la colonisation» autour de la marchandise, qui au début semble "économiciste ", permet de reprendre le fondement marxiste de la marchandise comme "abstraction réelle ( Marx, 1985). Ainsi, même si la production de denrées tropicales, sous le monopole métropolitain, par l'esclavage et 
sur de grandes étendues de terre était radicalement différente des manufactures anglaises, réalisées dans des industries privées et avec des salariés, toutes deux devraient devenir pareilles dans le marché, en provoquant, face à l'échange et à la production pour l'échange, l'effacement social de leurs conditions sociales de (re)production. Un tel mécanisme d'abstraction réitéré dans la pratique caractérise le substrat de l'impersonnalité du marché. La constitution et la généralisation des pratiques médiatisées par les marchandises et, ainsi, par le marché, seraient alors compatibles, dans leur impersonnalité et abstraction, à la dissémination d'un certain individualisme appuyé sur la tendance à la reconnaissance des travailleurs en tant que citoyens. Un moment important de cette reconnaissance concerne leur droit à la propriété de leurs propres corps et la capacité productive qui y est comprise. De cette façon, le travailleur libre qui se loue sur le marché constitue une catégorie sociale moderne en formation, ce qui ne devait se généraliser et être socialement reconnu dans quelques réalités de la fin du XVII ${ }^{\mathrm{e}}$ (Franco, 1984). Néanmoins, en raison de la simultanéité de cette constitution avec la réalité sociale, les liens connectant de tels " mondes » apparemment séparés ne se limitaient pas à des échanges immédiats.

L'« exclusif métropolitain» se posait alors en tant que mécanisme d'accumulation montrant bien la position inégale entre Métropole et Colonie, ainsi que le monopole sur le corps du travailleur esclave qui rendait évidente la position totalement inégale et hiérarchique entre maître et esclave. Sur ce point, il n'y a toujours pas de différence substantielle dans la situation d'indigènes et africains, ce qui devait aller dans le sens d'une «question raciale» (Vainer, 1984; Quijano, 2005) construite, d'une part, par la "guerre des barbares" rendant les populations esclaves et "pacifiant" les terres indigènes (Puntoni, 2002) et, d'autre part, par la conformation de l'esclavage négrier comme un mécanisme important de cumulation de capital pour la métropole (Alencastro, 2005). Ou alors, selon Gilberto Freyre (2000), une colonisation menée par "l'initiative privée épée en mains". Cependant, une telle initiative se trouverait attachée aux intérêts du gouvernement métropolitain (Faoro, 1989). La conquête forcée $\mathrm{du}$ territoire et la reproduction de la Colonie mettaient sous lumière le rôle central de la violence pour mobiliser le travail sous force et pour réaliser le «sens profond de la colonisation ", l'accumulation de capital en Europe (Novais, 2005, p. 70). «En attendant, devient complète la connotation du sens profond de la colonisation: commerciale et capitaliste, c'est-à-dire, un élément constitutif du processus de formation du capitalisme moderne "

Dans la Colonie, un tel processus diverge du cas « classique » d'accumulation primitive, basé sur l'expulsion des paysans, sur la clôture de terres communales (enclosures), sur une législation punissant le vagabondage et sur la contrainte au travail industriel (Marx, 1985). L'initiative majeure de l'accumulation primitive anglaise était donc le land grabbing, l'appropriation privée de la base foncière menant à l'expropriation du peuple. Le terme land grabbing renvoie au changement rapide de propriété ou de domaine, pouvant concerner non seulement la terre, mais aussi des brevets et des market shares. Il est utilisé dans la langue portugaise comme synonyme d'accaparement, de propriété des étrangers ou d'expropriation, malgré la suggestion de S. Sauer et S. Borras Jr. (2016, p. 12-13) du terme «appropriation de terres » par sa portée et par l'éventail de possibilités qu'il permet, y compris celle d'un contrôle qui ne passe pas forcément par la propriété privée directe de la terre, renvoyant à des formes de control grabbing, comme suggéré par N. Peluso et C. Lund (2011). Il s'agit d'une littérature 
récente très étendue, pas toujours basée sur un fondement marxiste. Pourtant, le terme land grabbing, selon White et al. (2012, p. 621), a été tout d'abord conçu par K. Marx luimême, dans le chapitre cité ci-dessus sur «La prétendue accumulation initiale ». Nous revenons ainsi à cette référence pour concevoir le terme en question comme faisant partie de nombreuses initiatives dans le processus de monopolisation des moyens de production, en produisant simultanément la propriété du capital, de la terre et de la force de travail, sur un mode fétichiste, en tant que marchandises qui apportent à leurs propriétaires des revenus distincts et apparemment autonomes. En particulier, l'expropriation de la population par rapport à la terre (moyen de production non produit) est caractéristique de ce qui s'est passé dans le cas anglais discuté par Marx.

Selon Franco (1984), dans la Colonie, l'expropriation significative du "peuple» s'exprimait par rapport à son propre corps, constituant ainsi une production de marchandises envoyées sur le marché externe au lieu d'être consommées à l'intérieur par un "peuple " confronté à de terres abondantes et au libre accès potentiel aux moyens de production. A contrario, était alors établie une espèce de labor grabbing, soutenant par l'esclavage la séparation entre terre et travail au profit du capital. À celui-ci était attribuée une connotation encore plus néfaste dans la mesure où le trafic négrier était l'une des voies les plus rentables pour la colonisation (Novais, 2005), bloquant la possibilité d'autonomisation des colons portugais face à la Métropole par l'esclavage d'indigènes pour l'occupation de la base foncière. On organisait l'approvisionnement d'esclaves venus d'Afrique, rendant évidente une relation intercoloniale dans l'Atlantique Sud (Alencastro, 2005), produisant des marchandises en régions tropicales organisées comme des « archipels » (Seabra \& Goldenstein, 1982), liés au commerce métropolitain, et entre eux de façon partielle. On estime que, entre le $\mathrm{XVI}^{\mathrm{e}}$ et le XIX ${ }^{\mathrm{e}}$ siècles, environ 11 millions d'africains ont été apportés en Amérique comme esclaves, dont $40 \%$ vers la Colonie portugaise (Lovejoy, 2002, p. 368), malgré le fait que des estimations plus récentes montrent un nombre encore plus élevé, d'environ 12,5 millions et $45 \%$ respectivement $^{1}$.

De nombreux méandres historiques amèneront à la mise en question de cette forme de colonisation de l'Ancien Régime, basée sur le labor grabbing ${ }^{2}$. Nous avons mentionné brièvement ci-dessus la généralisation du "préjugé d'humanité » (Marx, 1985, p. 65) renforcée par les salariés en Angleterre et par les idéaux révolutionnaires du libéralisme du XIX ${ }^{e}$. Cette généralisation mènerait encore à la mise en question du blocage de la liberté explicite dans l'esclavage. Aboutissant à la chute de «l'exclusif métropolitain ", au moment de l'arrivée de la famille royale au Brésil, en 1808, et ultérieurement à l'ouverture des ports, suivie de l'Independence en 1822, cette mise en question devrait imposer en effet une nouvelle dynamique à la colonisation.

16 La division entre territoire colonial et État absolutiste métropolitain posait la question d'une gestion interne du territoire national. Pour Moraes (1998), à partir du XIX siècle, le territoire de la colonisation est conçu progressivement comme territoire de l'État national, considérant les "sertões $\|^{3}$ et de ce qui n'a pas encore été approprié comme des "réserves foncières", pour la territorialisation future. Il a été alors possible de penser à une "longue liquidation du colonial» (Prado Jr., 1969), ou à une « internalisation de la métropole » (Dias, 2009) progressive, impliquant un autre regard sur le territoire. Ainsi, reprenant l'interprétation qui donnait du sens à la colonisation par la production de marchandises tropicales issues de grandes exploitations avec du travail esclave et le monopole métropolitain, se serait produite la chute d'un premier 
pilier avec l'Indépendance, mais il faudrait encore penser aux conditions de production de marchandises dans le territoire national et poser la question de savoir si leur destination serait le marché extérieur.

\section{La région entre le labor grabbing et le land grabbing}

17 Pour expliquer la « voie anglaise » de développement de l'agriculture, la modernisation s'est appuyée, comme nous l'avons dit, sur l'expropriation face au land grabs imposant la séparation entre travailleur et terre, menant à la « libération » des deux, auparavant fusionnés dans la catégorie de paysans (Marx, 1986). Cette libération "négative » du travailleur par rapport aux moyens de production devenait un présupposé de sa liberté " positive " à aller et venir, de choisir où et avec qui travailler, parce que, comme nous l'avons vu, celui-ci garderait la propriété de sa force de travail, la louant simplement dans le marché (Gaudemar, 1977). De cette façon, terre captive s'articule à travail libre. De façon inverse, dans la colonie, l'abondance de terres occupables avait impliqué l'esclavage. Au cours du XIX siècle s'est posée la question de comment accumuler du capital avec la critique croissante des pratiques de labor grabbing dans des réalités où la dynamique même du capitalisme n'avait pas été suffisante pour la réalisation du land grabbing de tout le territoire.

Dans ce débat surgit à l'époque un rudimentaire aménagement d'État. Il s'organise à partir de l'expérience frustrée de capitalistes anglais essayant de reproduire leurs entreprises dans les colonies, racontée par Wakefield (2001), devenue la proposition d'une «théorie de la colonisation moderne " (Marx, 1985, chap. 25). Il s'agissait de voir comment éviter la perte des investissements due au manque de travailleurs, qui préféraient d'occuper les terres à la frontière plutôt que de s'offrir sur le marché. Le manque relatif de travailleurs impliquait l'augmentation des salaires et la diminution de la plus-value, en limitant l'accumulation. La solution suggérée par Wakefield était fondée sur l'action de l'État suivante : promulguer une législation foncière, créant de façon artificielle la raréfaction de terres. Ainsi l'État se verrait obligé de régulariser les propriétés, déclarant les terres restantes comme appartenant à l'État pour les revendre dans le but de constituer un fonds public d'incitation à l'immigration de travailleurs européens. Le modèle de colonisation systématique de Wakefield a été assez discuté au Parlement brésilien au cours des années 1840, aboutissant à la Loi $n^{\circ} 601 / 1850$ et à l'interdiction de la traite (Costa, 1998 ; Lima, 1990).

Même s'il était inspiré des Homestead Acts, décrétés aux États-Unis dans les décennies suivantes et responsables directs de l'organisation de la « course vers l'Ouest » dans le pays (Costa, 1999), le plan de colonisation de Wakefield, implanté au Brésil, serait confronté à une réalité dans laquelle l'État n'a pas procédé de fait à la régularisation et à la vente des terres. Il revenait à l'Église catholique d'enregistrer les possessions, dans un premier temps. Ultérieurement, les colonels étaient chargés de recevoir ou de contrôler les nominations des notaires, ce qui renforçait leur pouvoir local et réglait toute dispute foncière au bénéfice des alliés (Boechat, 2014).

Le « colonélisme » émerge alors comme la facette contradictoire de la réalisation de la colonisation systématique. Pour le Brésil, en particulier, la Guarda Nacional apparaît comme la forme institutionnelle aussi bien de l'État national en formation que du pouvoir régional. Fondé en 1831, ce bras civil de l'Armée représentait, par moyen de concessions de brevets, la territorialisation de l'État même, malgré la délégation de son 
autorité à de leaders locaux, de grands propriétaires qui devenaient des colonels, et qui pouvaient recruter des « soldats ». Si la définition de Weber sur l'État moderne exige le monopole d'État de la violence, ce monopole apparemment "sous-traité " à une oligarchie nationale était juste. Une telle institution semble marquer des formes singulières de reproduction du capital et de la lutte de classes, où économique et politique se fondent (Oliveira, 2008, p. 29). Suite à la proclamation de la République, en 1889, des mécanismes similaires de pouvoir régulant les disputes territoriales seraient appliqués dans la centralisation de votes par les colonels locaux, en remontant les tensions et les disputes régionales à l'échelle nation. C'est pourquoi la Révolution de 1930 a cherché à imposer le désarmement des colonels, la centralisation et le renforcement de l'Armée nationale, ainsi qu'une institutionnalisation d'une justice électorale autonome, entre autres changements (Leal, 1976; Faoro, 1989). La Guarda Nacional peut donc être comprise comme une forme reconnue et institutionnelle de domination territoriale de l'État brésilien jusqu'à la fin de la Première République (1889-1930), persistant jusqu'à 1924 et n'étant désarmée que par les troupes du Président Getúlio Vargas.

21 L'expansion, dans le sud-est brésilien, des cultures de café s'est produite dans ce contexte. Tout d'abord dans la vallée du Paraíba, avec du travail esclave originaire de la traite et, après 1850, avec les esclaves achetés de régions esclavagistes décadentes dans le Nord, pour se déplacer ensuite vers l'ouest de São Paulo avec du travail libre recruté surtout en Europe méditerranéenne, et constituant une culture de café qui s'étendait mais se différenciait, du point de vue social et de la production, en deux régions qui s'opposaient (Beiguelman, 2005). Le Parlement de la province de São Paulo a approuvé, en 1862, l'apport de subsides à des investissements en voies ferrées, en même temps qu'il soutenait l'extermination indigène ${ }^{4}$ dans l'ouest de province, rendant viable l'expansion du front pionnier (Monbeig, 1998), en payant le voyage d'immigrants européens venus pour travailler dans les cultures de café ${ }^{5}$, exporté alors vers les pays industrialisés, encourageant ainsi le travail industriel.

Il faut souligner la question de la simultanéité du manque de bras pour la culture de même que le blocage, ou l'entrave à l'expansion, du front pionnier du café par la question indigène, mais il y avait d'autres points obscurs. Tandis que le colonélisme imposait son ordre à l'occupation des terres, en orientant les voies ferrées vers ses propriétés et les villages associés, au détriment d'autres, étaient établies des relations avec le capital financier et industriel anglais par l'intermédiaire de commissaires et des sociétés ferroviaires et exportatrices (Boechat, 2014). Les liens entre la région et le marché global deviennent évidents quand nous observons la mobilisation du capital et du travail dans les économies industrialisées (Harvey, 2006).

Effectivement distinct de l'esclavage, le système de travail du colonat pour la culture postcoloniale du café ne pourrait pas être vu comme un simple travail salarié, sauf par des analyses controversées. La gestion d'un certain nombre de pieds de café restait sous la responsabilité de l'immigrant et de sa famille, bien qu'il leur ait été permis, outre la plantation de café, de produire des aliments pour eux-mêmes et pour la vente. Ce contrat donnait droit à un salaire monétaire régulier et à une proportion de la récolte, outre l'accès à une maison et à une partie d'un pâturage à l'intérieur de la ferme. C'est donc une des formes sociales contradictoires dans lesquelles travail et capital ne se séparaient pas, tout en confondant les revenus (revenus, salaire et profit) et se 
reproduisant comme travail (ou exploration) familiale non complètement individualisé, même organisé par le père dans une relation patriarcale.

De nombreuses relations sociales de production prennent de formes particulières, comme dans l'extraction de caoutchouc en Amazonie (Gonçalves, 2005), la production de canne-à-sucre dans le Nord-est (Oliveira, 2008), les prospecteurs de la Chapada Diamantina (Toledo, 2008), ou les cowboys de l'élevage qui se disséminait vers les sertões (Leite, 2015), même s'ils ressemblaient à certains éléments du colonat. Le travail sur la frontière agricole brésilienne entre les décennies 1870 et 1960 semble être, alors, un travail libre ayant accès aux moyens de production, dont les termes étaient négociés avec les colonels locaux.

Nous suggérons donc de penser la région en prenant en compte de telles particularités comme une partie du processus de territorialisation des rapports sociaux de production dans lesquels les pratiques d'esclavage ont été progressivement bloquées et dans lesquelles la liberté d'insertion contradictoire du travailleur était relativisée par l'intermédiaire du culturel, de la famille ou de la politique. Celle-ci a constitué le mécanisme central pour la mise en question de la pratique de la colonisation auparavant appuyée sur l'esclavage, permettant et encourageant ainsi la dispute politique qui devrait aboutir à l'abolition de l'esclavage, en 1888, et à la proclamation de la République, en 1889. La transition du labor grabbing au land grabbing au Brésil, toutefois, ne serait pas totale jusqu'à la modernisation de l'agriculture post-guerre. En même temps, un processus renforcé d'industrialisation et d'urbanisation ${ }^{6}$ caractérise une transformation sociale considérable.

\section{Planification d'État et émergence de la question régionale}

Le cas d'une région particulière nous permet de reprendre la proposition d'Oliveira (2008) sur l'enfermement politique de la région et de sa rupture ultérieure. Différente $d u$ « recrutement » d'esclaves et d'hommes libre à l'étranger, un apport extérieur de travailleurs incorporés aux relations régionales, la possibilité d'un commerce interprovinces d'esclaves post-1850 montrait une fermeture relative des régions fournisseuses, ce qui est souligné en pointant vers des liaisons et tensions entre les échelles diverses d'analyse. Suite à l'établissement des relations sociales particulières, ce recrutement devrait se passer, majoritairement, à l'intérieur même des régions. La rupture de cette "réserve $»^{7}$ est une pré-condition pour l'établissement du marché interne, en réarticulant les « archipels ». Oliveira, même s'il concède à la géographie la filiation conceptuelle du terme, se confronte enfin à la tension due à l'appropriation de ce terme par la planification de l'État, établie en tant qu'aménagement régional :

"L'aménagement surgit ici comme une 'forme' d'intervention de l'État sur les contradictions entre la reproduction du capital à l'échelle national et régionale, 'assumant l'allure de conflits inter-régionaux'; l'aménagement n'est pas, donc, la présence d'un État médiateur, mais, au contraire, la présence d'un État capturé ou non par les formes plus avancées de la reproduction du capital pour forcer le passage vers une homogénéisation ou, comme décrit par la littérature sur la planification nationale, vers 'l'intégration nationale' "(Oliveira, 2008, p. 149).

Le structuralisme fondant cet aménagement a quantifié des données prédéterminées afin de montrer des écarts et de proposer des interventions dans les régions. Cet 
auteur, dans le refus de prendre le rôle de modernisation de l'État en tant que " neutre », focalise l'existence de conflits entre des formes d'accumulation, l'État étant ainsi l'instrument d'hégémonie. Nous pouvons donc indiquer que l'apparition d'une bureaucratie constitue une partie nécessaire à la constitution d'un État qui s'autonomise du capital, avec des instances non totalement ni directement déterminées par celui-ci, ni dans le seul but de l'accumulation (voir Kurz, 1998). La formation de techniciens et de cadres pour les secteurs de l'État montre un développement logique d'une différence de la forme régionale, basée sur des «engagements » des colonels (Leal, 1976).

Dès lors, l'université avance pari passu vers cette (trans) formation de l'État. La formation des conditions pour établir une bureaucratie qui représente l'État est un élément important du passage vers une autre forme de domination sociale légitime autre que le commandement (Boechat, 2014 ; Leite, 2015).

29 Suite à ce processus, la "région » est re-signifiée par l'État et ses instances, s'orientant vers la conformation aujourd'hui naturalisée des régions intégrées dans l'aménagement national - Nord, Sud, Centre-Ouest, Sud-est et Nord-est - originaires du processus de territorialisation et $\mathrm{du}$ processus d'intégration nationale qui en résultante, l'assimilant et le modifiant. Dans une tentative d'intervention, les différences découlant des relations sociales de productions spécifiques semblent être réduites, de façon fétichiste et tendancieuse, à des inégalités d'allocation de facteurs, en introduisant la soi-disant question régionale dans la technocratie qui oriente de façon supposée une telle intervention. Néanmoins, elle n'en reste pas là.

La période entre 1930 et 1970 est alors marquée par un conflit qui montre l'appropriation partielle des excédents pour l'industrialisation, sans une rupture totale d'avec les relations de production jusqu'alors en vigueur (Stolcke, 1986). D'une part, on permettait la constitution progressive du marché national de travail, au bénéfice de l'industrialisation du Centre-sud; d'autre part, les régions se resituaient de façon fonctionnelle permettant le maintien de l'accumulation par l'approvisionnement en aliments et en matières-premières, en augmentant la main-d'œuvre, et en créant du marché, du transfert et de la réception de capitaux (Barros de Castro, 1969, p. 94-96). La modernisation tardive était ainsi stimulée, dans le sens de favoriser l'industrialisation nationale dans une course pour atteindre les paliers de productivité des économies centrales. La détérioration partielle des relations politiques économiques qui l'organisaient permet une orientation d'une partie de l'accumulation régionale vers la planification nationale de l'industrialisation (Oliveira, 1977). D'autre part, il faudrait une conjoncture internationale favorable pour permettre une exploration de capitaux promouvant un développement effectif de l'industrie (Mello, 2008).

31 Nous suggérons ainsi que "l'intégration nationale ", ayant comme "téléologie " (Costa, 2013) la rupture entre les relations de pouvoir et la transformation des formes particulières de reproduction des relations sociales de production de la région, doit formuler au niveau (national) de discussion une question régionale. Celle-ci se développe vers une question migratoire et une question foncière, devant composer la question agraire et la question urbaine des disputes pour les orientations et les sens de la modernisation. Nous reprenons ici la suggestion de Vainer (1984), pour qui s'impose progressivement une perspective qui s'adresse à l'État comme un " médecin de la société "qui cherche à guérir des «pathologies portées par l'organisme social ». Celles-ci encourageant des discours officiels divers, toujours autour de «l'occultation-déplacement » des causes 
réelles des "pathologies». "Que la question raciale puisse donner lieu à la question régionale, que la question des bras pour l'agriculture soit dépassée par la question urbaine, voici des phénomènes qui font signe de l'apparition de nouvelles formes de contrôle du travail dans l'espace» (Vainer, 1984, p. 42).

\section{Conclusion}

Nous rappelons qu'Oliveira (2008/1987) avait cité une « homogénéisation » des régions dans des « zones de localisation différentielle » nationalement intégrées, ayant subi des critiques dures dans la géographie brésilienne pour ne pas avoir observé le remplacement constant des différences dans la production de l'espace (Lencioni, 1999). Néanmoins, il faut observer la logique sous-jacente à sa formulation, qui ne niait pas la différence dans l'homogénéité, celle-ci auparavant étant marquée par des inégalités. Finalement, qu'est-ce qui pourrait être homogène? L'«ouverture» de la région implique l'autonomisation entre l'économique et le politique et donc la mobilisation du travail. Pour Oliveira (2008), le «sens profond » du processus peut être pointé comme l'emploi, défini dans l'immigration de travailleurs venant des régions pour l'industrialisation et l'urbanisation nationales. Toutefois, il avait affirmé que l' «accumulation primitive » était structurée de façon nationale par «l'élaboration de banlieues" (Oliveira, 2003, p.43), ce qui implique un double sens d'un travailleur installé dans les banlieues urbaines et dans l'expansion du capitalisme vers la banlieue productrice, au moyen du front pionnier, en reproduisant les conditions " primitives » d'accumulation et en recomposant les relations de travail non salariées.

Nous avons suggéré, pourtant, qu'une région spécifique était attachée à une colonisation systématique dans laquelle des limites à sa "mobilité parfaite » du travail se situaient dans la pratique, tandis que, contrairement, leur fonctionnement même favorisait la mobilité. Nous avons vu que la colonisation systématique a soutenu la promulgation de lois et de politiques d'immigration, constituant un surpeuplement relatif permettant l'accumulation de capital dans le contexte de l'existence de frontières ouvertes. De ce mode, nous comprenons l'intention latente de permettre l'emploi dans cette législation et dans la systématisation de la pratique de colonisation ; cette intention est "tordue par la réalité » (Faoro, 1989) où elle a été employée. Le colon réunit les catégories du capital (terre, travail et capital) et sa "modernisation " implique l'autonomisation de ces catégories en différentes personas. Nous proposons ici une compréhension de la modernisation retardataire dans sa facette régionale qui refuse le dualisme explicite, par exemple, chez Oliveira même (2003), une fois que nous concevons les relations dans la région comme éminemment modernes, et non pas féodales, traditionnalistes ou primitives. La modernisation est prise ici comme processus d'autonomisation du capital. Le salaire du travailleur est une voie possible pour que cela puisse se faire, sans être la seule. La région a été un moment de la territorialisation du capital dans une réalité donnée, mais elle ne suivra pas rétablie, de façon à ce que l'urbanisation, l'industrialisation et la financiarisation doivent conférer de conditionnements nouveaux et critiques à la reproduction sociale dans la campagne et dans la villes.

34 Le parcours final a été marqué par les législations promulguées dans les années 1960. Le Statut du Travailleur Rural (Loi 4.212. de 1963), et le Statut de la Terre (Loi 4.504 de 1964) sont considérés comme une relecture nationale de la territorialisation de l'État 
national et du capital, ayant jusqu'alors comme paramètre les politiques de colonisation systématique. Si la Loi des Terres (Loi 601 de 1850) synthétise, comme nous l'avons vu, un projet de colonisation basé sur le monopole de classe sur la terre (land grabbing), réunissant toute une série de mesures pour bloquer l'esclavage (labor grabbing), les - Loi Eusébio de Queiroz (Loi 581 de 1850), Loi du Ventre Libre (Loi 2.040, de 1871), Loi du Sexagénaire (Loi 3.270, de 1885) et Loi Áurea (Lei 3.353 de 1888) -, les nouvelles législations, des années 1960 , n'ont pas réfuté cette qualité fondamentale de l'accumulation de capital dans l'agriculture moderne, mais se sont orientées contre des éléments de la colonisation systématique, surtout concernant les formes régionales de travail. La règlementation du partenariat agricole et, donc, de l'accès à la terre et d'autres modalités de rendement du travail agricole, au sein du Statut de la Terre, suggèrent un aménagement en faveur de l'emploi, un aspect qui a contribué à l'expulsion massive de travailleurs agricoles dans les fermes (Silva, 1999), même si le Statut règlemente également la Réforme Agraire et des politiques de colonisation. La question régionale, pensée ici en termes critiques et de formation, semblait donner lieu, pourtant, à une question agraire tendue, toujours loin d'être envisagée.

Nous suggérons alors que ce qui était en jeu au début de la période dictatoriale (1964-1985) était la fin de la quadrature historique de la colonisation systématique dans les termes de la région. Il faut donc penser combien cet aménagement a été bien réussi et combien il a été mis en place en affirmant ce nouveau sens ou bien en le contredisant.

\section{BIBLIOGRAPHY}

Alencastro L. F. de, 2005. O trato dos viventes: formação do Brasil no Atlântico Sul. São Paulo, Companhia das Letras., $544 \mathrm{p}$.

Beiguelman P., 2005. A formação do povo no complexo cafeeiro - aspectos políticos. São Paulo, Edusp., $280 \mathrm{p}$.

Boechat C. A., 2014. O colono que virou suco: terra, trabalho, Estado e capital na modernização da citricultura paulista. São Paulo, Universidade de São Paulo, 569 p.

Boechat C. A., 2009. Região do colonato: mobilização do trabalho e autonomização do capital na área de Olímpia (1857-1964) do Oeste Paulista. São Paulo, Universidade de São Paulo, 311 p.

Brant V. C., 1976. Do colono ao bóia-fria: transformações na agricultura e constituição do mercado de trabalho na Alta Sorocabana de Assis. Estudos CEBRAP, n 19, p. 37-91.

Buainain A. M., Alves E., Silveira J. M., Navarro Z, 2014. Sete teses sobre o mundo rural brasileiro. In Buainain A. M., Alves E., Silveira J. M., Navarro Z. (org.), o mundo rural no Brasil do século 21. Brasília, Embrapa, p. 1 159-1 186.

Carneiro M. J., 2008. Rural como categoria de pensamento. Ruris, vol. 2, nº 1.

Castro A. B., 1969. Sete ensaios sobre a economia brasileira. Rio de Janeiro, Forense, 193 p. 
Costa E. V. da, 1999. Da monarquia à república - Momentos decisivos. $7^{a}$ ed., São Paulo, Fundação Editora da UNESP, $490 \mathrm{p}$.

Costa E. V. da, 1998. Da senzala à colônia. 4ª . ed., São Paulo, Fundação Editora da UNESP, 560 p. Costa F., 2013. Sete teses sobre o mundo rural brasileiro: antíteses. Revista da ABRA, edição especial, p. 45-60.

Dias M. O. S. L., 2009. A interiorização da metrópole e outros estudos. $2^{\text {a }}$ ed., São Paulo, Alameda, 325 p. Faoro R., 1989. Os donos do poder: formação do patronato político brasileiro. $8^{\mathrm{a}}$ ed., São Paulo, Globo, $750 \mathrm{p}$.

Franco M. S. de C., 1997. Homens livres na ordem escravocrata. São Paulo, Fundação Editora da UNESP, $253 \mathrm{p}$.

Franco M. S. de C., 1984. Organização social do trabalho no período colonial. In Pinheiro P. S., Trabalho escravo, economia e sociedade. São Paulo, Paz e Terra, p. 143-192.

Freyre G., 2000. Casa-Grande \& Senzala - introdução à história patriarcal no Brasil. Rio de Janeiro, Record, $66 \mathrm{p}$.

Furtado C., 2000. Formação econômica do Brasil. São Paulo, Brasiliense, 276 p.

Gaudemar J. P. de, 1977. Mobilidade do trabalho e acumulação do capital. Lisboa, Estampa, 405 p. Gonçalves C. W. P., 2005. Amazônia, Amazônias. Rio de Janeiro, Contexto, 178 p.

Harvey D., 2005. A produção capitalista do espaço. São Paulo, Annablume, 249 p.

Harvey D., 2001. O novo imperialismo. São Paulo, Loyola, 201 p.

Holanda S. B. de, 1995. Raízes do Brasil. São Paulo, Companhia das Letras, 256 p.

IBGE, 2000. Brasil 500 anos de povoamento. brasil500anos.ibge.gov.br.

IBGE, 2010. Censo de 2010 - resultados. censo2010.ibge.gov.br.

Jackson L. C., 2007. Tensões e disputas na sociologia paulista (1940 - 1970). Revista Brasileira de Ciências Sociais, vol. 22, nº 65, p. 33-49.

Kurz R., 1998. A falta de autonomia do Estado e os limites da política. In Kurz R., Os últimos combates. Petrópolis, Vozes, p. 91-118.

Kurz R., 2000. O colapso da modernização - Da derrocada do socialismo de caserna à crise da economia mundial. Rio de Janeiro, Paz e Terra, 231 p.

Leal V. N, 1976. Coronelismo, enxada e voto - O município e o regime representativo no Brasil. São Paulo, Alfa-Ômega, 276 p.

Leite A. C. G., 2015. 0 campesinato no Vale do Jequitinhonha: da sua formação no processo de imposição do trabalho à crise da (sua) reprodução capitalista. São Paulo, Universidade de São Paulo, 762 p.

Lencioni S., 1999. Região e geografia. São Paulo, Editora da Universidade de São Paulo, 215 p.

Li T. M., 2011. Centering labor in the land grab debate. The Journal of Peasant Studies, vol. 38, $n^{\circ} 2$, p. 281-298.

Lovejoy P. E., 2002. A escravidão na África: uma história de suas transformações. Rio de Janeiro, Civilização Brasileira, 467 p.

Lima R. C., 1990. Pequena história territorial do Brasil: Sesmarias e terras devolutas. São Paulo, Secretaria de Estado da Cultura, 112 p. 
Lipietz A., 1988. O capital e seu espaço. São Paulo, Nobel, 209 p.

Luxemburg R. Acumulação de capital: contribuição ao estudo do imperialismo. São Paulo, Nova Cultural, $418 \mathrm{p}$.

Martins J. S., 1998. O cativeiro da terra. São Paulo, Hucitec, 157 p.

Marx K., 1985. O Capital - crítica da Economia Política. V. I. São Paulo, Nova Cultural, 301 p.

Marx K., 1986. O Capital - crítica da Economia Política. V. IV. São Paulo, Nova Cultural, 335 p.

Mello J. M. C. de., 2009. 0 capitalismo tardio. Campinas, Ed. FACAMP e EdUnesp, 150 p.

Monbeig P., 1998. Pioneiros e fazendeiros de São Paulo. São Paulo, Hucitec/Polis, 392 p.

Moraes A. C. R. de., 2003. Geografia: pequena história crítica. São Paulo, Annablume, 130 p.

Moraes A. C. R. de.1998. Ideologias geográficas: espaço, cultura e política no Brasil. São Paulo, Hucitec, $156 \mathrm{p}$.

Novais F., 2005. Portugal e Brasil na crise do antigo sistema colonial (1777-1808). São Paulo, Editora Hucitec, $420 \mathrm{p}$.

Oliveira A. U., 1987. Modo capitalista de produção e agricultura. São Paulo, Ática, 88 p.

Oliveira F. de, 2003/1972. Crítica à razão dualista; O ornitorrinco. São Paulo, Boitempo Editorial, $150 \mathrm{p}$.

Oliveira F. de, 1977. Economia da dependência imperfeita. Rio de Janeiro, Graal, 159 p.

Oliveira F. de, 2008/1987. Elegia para uma re(li)gião: Sudene, Nordeste. Planejamento e conflito de classes; Noiva da Revolução. São Paulo, Boitempo, 278 p.

Oliveira F. de, 2006. O vício da virtude - autoconstrução e acumulação capitalista no Brasil. Novos Estudos, n. 74, São Paulo, p. 67-85.

Oliveira F. de, 1993. Questão regional: hegemonia inacabada. Estudos Avançados, vol. 7, n 18, a. 03, São Paulo, p. 43-63.

Peluso N., Lund C., 2011. New frontiers of land control. The Journal of Peasant Studies, vol. 38, $\mathrm{n}^{\circ} 4$, p. 667-681.

Prado JR. C, 1979. A questão agrária no Brasil. São Paulo, Brasiliense, 188 p.

Prado JR. C, 2000. Formação do Brasil contemporâneo: Colônia. São Paulo, Brasiliense, 390 p.

Prado JR. C, 1969. História econômica do Brasil. São Paulo, Brasiliense, 352 p.

Puntoni P., 2002. A Guerra dos Bárbaros: Povos Indígenas e a Colonização do Sertão Nordeste do Brasil, 1650-1720. São Paulo, HUCITEC/Edusp/Fapesp, 324 p.

Quijano A. 2005. Colonialidade do poder, eurocentrismo e América Latina. In Lander E., A colonialidade do saber: eurocentrismo e ciências sociais. Perspectivas latino-americanas. Buenos Aires, CLACSO, p. 107-129.

Rangel I. M., 1986. A questão da terra. Revista de Economia Política, vol. 6, n 4, p. 71-77.

Sauer S., Borras JR. S. M., 2016. "Land grabbing" e "green grabbing": uma leitura da "corrida na produção acadêmica" sobre a apropriação global de terras. Campo-Território - Revista de Geografia Agrária, Edição Especial, p. 6-42.

Schwarz R., 2000. Um mestre na periferia do capitalismo: Machado de Assis, Rio de Janeiro, Duas Cidades/Editora 34, 256 p. 
Seabra M., Goldenstein L., 1982. Divisão territorial do trabalho e nova regionalização. Revista do Departamento de Geografia, ${ }^{\circ}$ 1, p. 21-47.

Silva M. A. M., 1999. Errantes do fim do século. São Paulo, Fundação Editora da UNESP, 370 p.

Sorre M., 1984. Migrações e mobilidade do ecúmeno. In Sorre M., Max Sorre, Geografia, São Paulo, Ática, p. 124-139.

Stolcke V., 1986. Cafeicultura - homens, mulheres e capital (1850-1980). São Paulo, Brasiliense, 410 p.

Toledo C. A., 2008. A região das lavras baianas. São Paulo, Universidade de São Paulo, 245 p.

Toledo C. A., Boechat C. A., 2012. Mobilização do trabalho e o estudo da região. Boletim Campineiro de Geografia, vol. 2, n³ 3, p. 448-464.

Vainer C. B., 1984. Trabalho, espaço e estado: questionando a questão migratória. Cadernos IPUR, vol. $1, \mathrm{n}^{\circ} 1$, p. 6-43.

Wanderley M. N. B. 0 mundo rural como um espaço de vida - reflexões sobre a propriedade da terra, agricultura familiar e ruralidade. Porto Alegre, UFRGS Editora, $329 \mathrm{p}$.

Wakefield E. G., 2001. A View of the Art of Colonization. Kitchner, Batoche Books, 154 p.

White, B., Borras Jr. S., Hall R, Scoones I., Wolford, W., 2012. The new enclosures: critical perspectives on corporate land deals. The Journal of Peasant Studies, vol. 39, n 3-4, p. 619-647.

\section{NOTES}

1. slavevoyages.org

2. De ce mode, tandis que les pratiques de land grabbing séparaient de force la population des champs de leurs moyens de vie, mobilisant le travailleur, ne lui donnant que sa propre force de travail à négocier "librement" en échange d'un salaire, les pratiques de labor grabbing, d'autre part, expropriaient la propriété de la force de travail du travailleur lui-même, l'empêchant de prendre "libre" possession des terres relativement disponibles dans le contexte colonial. Ce qui révèlerait, pour Marx (1985, chap. 25), le secret de la liberté du travailleur, contraint à des choix sous la forme de marchandises, sans avoir le choix de ne pas travailler, et ainsi de ne pas valoriser la valeur. Nous pouvons alors centrer le travail au débat sur land grabbing, comme le fait Li (2011), en présentant, de notre côté, la discussion sur l'esclavage colonial non pas comme mode de production à part ou comme un archaïsme, mais comme une espèce de radicalisation du fondement de l'accumulation de capital.

3. Terres non occupées, équivalent du "bled", "outback" ou "wilderness" (NdT)

4. Des données de la FUNAI (unai.gov.br) montrent l'existence, au XVI ${ }^{\mathrm{e}}$, d'environ un million d'indigènes à l'intérieur de la Colonie, à côté d'environ 2 millions vivant sur le littoral, tandis que l' IBGE estime qu'à São Paulo il y aurait environ 146000 indigènes de 8 groupes distincts. En 1957, la population indigène locale a atteint son niveau le plus bas, ayant autour de 70000 individus, dont 65000 en province, selon la FUNAI, une augmentation significative de cette population ayant été constatée (817000 indigènes comptés au Recensement de 2010, dont 545000 en province et 41000 à São Paulo (IBGE, 2000 ; IBGE, 2010).

5. Entre 1822 et 1980, 5827237 immigrants sont débarqués au Brésil, selon les données de l'IBGE (2000), qui a enregistré à chaque période, de 1890 à 1900, de 1901 à 1920, et de 1920 à 1940, respectivement 1143902,1466388 et 1269270 entrées. Donc, à l'apogée de l'expansion de la culture du café 3879560 immigrants sont entrés officiellement au Brésil, dans une période de 50 ans, la plupart d'entre eux s'adressant aux cultures de café et vers les villes du Sud-est, une grande majorité ayant leur voyage payé par le gouvernement de São Paulo (Boechat, 2009). 
6. Ce n'est que dans les années 1960 que l'industrie dépasse l'agriculture en participation au PIB national et que la population urbaine dépasse la population rurale (IBGE, 2000).

7. La notion de "réserve" est basée sur une orientation nationale du mouvement vers l'industrialisation, car celle-ci renvoie à la conception d' "armée industrielle de réserve" (Marx, 1985, chap. 23), y compris la variante d'une paysannerie potentiellement liée à la classe des travailleurs urbains, gardant leur "mobilisation" comme "latente", en attente des demandes du capital. Harvey (2001) souligne la qualité d'un "élément tiers" de cette paysannerie au-delà des relations entre capitalistes et travailleurs dans la reproduction élargie du capital, en récupérant la théorie de Luxembourg sur le besoin d'incorporation de l'économie naturelle ou paysanne dans le but de retenir l'effondrement du capitalisme, même si Harvey (2001) adopte l'explication causale distincte de Luxembourg (1984) pour cet effondrement. Nous reconnaissons, ainsi, une certaine théologie du terme "réserve", développée de façons distinctes dans les explications courantes, mais nous refusons de penser l'unité paysanne et les relations internes de la région en tant qu'autonomes, en préférant la perspective de l'autonomisation des catégories du capital (Marx, 1986, p. 47), comme quelque chose en cours et de façon contradictoire. Oliveira (2008) développe la perspective intéressante d'un conflit entre les formes du capital, exprimées en échelles géographiques.

8. Oliveira même (2006), dans un texte plus récent, affirme que la forme de reproduction de la banlieue urbaine par l'auto-construction, réduisant les coûts de reproduction de la force de travail, permettant la réduction de son propre salaire et, ainsi, promouvant l'industrialisation, s'était modifiée par la révolution technologique scientifique dans la ligne de production. Le rapport de la banlieue à l'industrialisation semble avoir été rompu et le travail informel et précaire deviendrait la règle, ce qui est partiellement reconnu par le sociologue.

\section{ABSTRACTS}

Cet article cherche à repenser la trajectoire historique de constitution des régions du Brésil par une double conceptualisation des procédures d'expropriation, centrées soit sur l'esclavage (labor grabbing), soit sur l'appropriation privée de la terre (land grabbing). De cette façon sont repris des auteurs classiques qui ont travaillé sur la formation nationale, tout en proposant alternativement d'aborder la modernisation par la notion d'autonomisation du capital. Nous souhaitons ainsi produire une approche théorique sur la constitution de la question agraire au Brésil comme découlant du processus qui, par l'intégration nationale, aborde la région comme un problème à régler.

\section{INDEX}

Mots-clés: région, colonisation systématique, labor grabbing, land grabbing, autonomisation du capital 


\section{AUTHORS}

\section{CÁSSIO ARRUDA BOECHAT}

Cássio Arruda Boechat, cassio.boechat@ufes.br, est Professeur au Département de Géographie de l'Universidade Federal do Espírito Santo (UFES). Il a publié récemment :

- Pitta F. T., Toledo C. A., Boechat C. A., 2016. A territorialização do capital da agroindústria canavieira paulista, ao longo do Proálcool (1975-1990). Revista Continentes (UFRRJ), vol. 5, p. 25-56.

\section{ANA CAROLINA GONÇALVES LEITE}

Ana Carolina Gonçalves Leite, carolinavecchia@gmail.com, est Professeur assistant et suit un post-doctorat au Programa de Pós-graduação em Geografia (PPGG) da Universidade Federal do Espírito Santo (UFES). Elle a publié récemment :

- Leite A. C. G., 2015. Expansão capitalista no Vale do Jequitinhonha: Pautas para a discussão do problema da acumulação primitiva. Revista Pegada Eletrônica (Online), vol. 16, p. 179-201.

\section{CARLOS DE ALMEIDA TOLEDO}

Carlos de Almeida Toledo, carlosdealmeidatoledo@gmail.com, est Professeur du Departamento de Geografia e do Programa de Pós-graduação em Geografia Humana (PPGH) da Universidade de São Paulo (USP). Il a publié récemment :

- Pitta F. T., Toledo C. A., Boechat C. A., 2016. A territorialização do capital da agroindústria canavieira paulista, ao longo do Proálcool (1975-1990). Revista Continentes (UFRRJ), vol. 5, p. 25-56. 